\title{
Changes in Platelet Counts and Thrombocytopenia Risk in Patients with Chronic Liver Disease with Different Etiologies Using Real-World Japanese Data
}

\author{
Manami Yoshida · Ryosuke Tateishi · Shinzo Hiroi · Masakazu Fujiwara • \\ Yoshitake Kitanishi · Kosuke Iwasaki · Tomomi Takeshima • \\ Ataru Igarashi
}

Received: July 28, 2021 / Accepted: November 29, 2021 / Published online: December 20, 2021

(C) The Author(s) 2021

\section{ABSTRACT}

Introduction: Thrombocytopenia, a common complication of chronic liver disease (CLD), adversely affects the treatment in patients

Supplementary Information The online version contains supplementary material available at https:// doi.org/10.1007/s12325-021-02008-x.

M. Yoshida $(\bowtie) \cdot$ S. Hiroi

Medical Affairs, Shionogi \& Co., Ltd., 7F, Tekko

Building, 1-8-2, Marunouchi, Chiyoda-ku, Tokyo

100-0005, Japan

e-mail: manami.yoshida@shionogi.co.jp

M. Yoshida · A. Igarashi

Graduate School of Pharmaceutical Sciences, The

University of Tokyo, 7-3-1 Hongo, Bunkyo-ku,

Tokyo 113-0033, Japan

R. Tateishi

Department of Gastroenterology, The University of

Tokyo Hospital, 7-3-1 Hongo, Bunkyo-ku, Tokyo

113-8655, Japan

M. Fujiwara · Y. Kitanishi

Data Science Department, Shionogi \& Co., Ltd., 3-1-8, Doshomachi, Chuo-ku, Osaka 541-0045, Japan

K. Iwasaki · T. Takeshima

Milliman, Inc., 8F, Kojimachi 1-chome Building, 1-6-2 Kojimachi, Chiyoda-ku, Tokyo 102-0083, Japan

\section{A. Igarashi}

Unit of Public Health and Preventive Medicine,

Yokohama City University School of Medicine, 3-9

Fukuura, Kanazawa-ku, Yokohama 236-0004, Japan requiring invasive procedures. Multiple pathophysiological mechanisms contribute to the development of thrombocytopenia; thus, its incidence could differ among CLD etiologies. We investigated the risk of decline in platelet counts and developing thrombocytopenia across different CLDs in a real-world Japanese setting.

Methods: A Japanese claims database including 25 million patients (April 2008-August 2018) was used. Patients with at least one CLD diagnosis were classified into nine mutually exclusive categories: hepatitis $\mathrm{B}$, hepatitis $\mathrm{C}$, hepatitis $B$ and $C$, unspecified viral hepatitis, autoimmune hepatitis, toxin/drug-induced hepatitis, alcoholic hepatitis, nonalcoholic steatohepatitis, and others. A random effects model was used to estimate the changes in platelet counts; proportional hazard analyses were used to examine factors associated with the incidence of thrombocytopenia based on the diagnosis. Patients with laboratory test data as variables were included in each analysis.

Results: The simulation included 68,536 patients. The mean values representing changes in the platelet count were significantly negative in the hepatitis $C$ patients and negative, though non-significant, in the hepatitis $\mathrm{B}$, toxin/druginduced hepatitis, alcoholic hepatitis, and nonalcoholic steatohepatitis patients. In the proportional hazard analysis, 708 of 22,728 patients had thrombocytopenia. The hazard ratio (HR) was significantly high for patients with hepatitis B (HR, 2.879; $p<0.001)$, 
hepatitis $\mathrm{C}(\mathrm{HR}, 1.876 ; p<0.001)$, and hepatitis $\mathrm{B}$ and $\mathrm{C}$ (HR, 2.992; $p<0.001)$.

Conclusion: A decreasing tendency in platelet counts was observed in most CLD etiologies, with hepatitis $C$ showing a significant decrease. The incidence of thrombocytopenia was mostly associated with hepatitis B and/or C. Further research is warranted to elucidate the discrepancy between the decline in platelet counts and thrombocytopenia diagnosis, considering the factors relevant to the diagnosis, such as the frequency of outpatient visits and CLD treatment.

Keywords: Cohort studies; Hepatitis; Hospital records; Liver diseases; Platelet count; Thrombocytopenia

\section{Key Summary Points}

Why carry out this study?

Thrombocytopenia is a common complication of chronic liver disease (CLD) and adversely affects the treatment in patients requiring invasive procedures

The incidence of thrombocytopenia may differ among CLD etiologies based on the variety of pathophysiological mechanisms associated with its development. Considering the possibility of the differences in the risk of thrombocytopenia and the recent changes in the distribution of patients among different etiologies, it is important to examine whether the development of thrombocytopenia differs among etiologies

This study investigated the risk of decline in the platelet counts and developing thrombocytopenia across different CLD etiologies in an entire clinical course in a real-world Japanese setting
What was learned from the study?

Platelet counts decreased with disease duration in most CLD etiologies; the decrease was particularly significant in patients with hepatitis C. The incidence of thrombocytopenia was more significant in patients with hepatitis B and/or C than in those with alcoholic hepatitis. The platelet count decreased in some of the other causes of CLD (toxin/drug-induced hepatitis, alcoholic hepatitis, and nonalcoholic steatohepatitis).

Furthermore, a significantly higher risk of thrombocytopenia was not observed between alcoholic hepatitis and the remaining diseases

A decline in platelet counts with disease duration was suggested in most CLD etiologies. The frequency of thrombocytopenia diagnosis was suggested to be different among various causes of CLD, even in patients with similar platelet counts, potentially owing to the differences in the frequency of outpatient visits and treatment of CLD

\section{INTRODUCTION}

Thrombocytopenia is a common complication of chronic liver disease (CLD) $[1,2]$. The significance of thrombocytopenia in patients with CLD is highlighted by the fact that these patients often require diagnostic and/or therapeutic invasive procedures. Furthermore, thrombocytopenia adversely affects the CLD treatment, namely by increasing the risk of bleeding and need for transfusions [2], thus delaying surgical procedures and potentially increasing the treatment costs.

It is known that thrombocytopenia is associated with CLD progression. Previous studies have reported that the prevalence rate of thrombocytopenia in patients with CLD is $15-70 \%$ and is lower in patients with compensated CLD and higher in those with end-stage liver disease [3]. In patients with cirrhosis or 
fibrosis, the prevalence rate of thrombocytopenia is reportedly $64-84 \%[4,5]$.

Multiple factors may contribute to the development of thrombocytopenia in patients with CLD. Historically, CLD-associated thrombocytopenia has been recognized as a consequence of hypersplenism and is due to an increased pooling and accelerated destruction of the platelets in the enlarged spleen secondary to portal hypertension $[4,6,7]$. Decreased production of thrombopoietin, mainly synthesized in the liver and involved in megakaryocyte growth and platelet production, may also result in thrombocytopenia in patients with CLD $[2,4,8]$. Moreover, suppression of platelet production in the bone marrow may be caused by certain underlying etiologies of liver disease $[2,4]$.

Furthermore, a difference in the risk of thrombocytopenia has been reported between some causes of CLD. A previous study comparing hepatitis $\mathrm{B}$ and $\mathrm{C}$ reported more severe thrombocytopenia in hepatitis $C$ for the same grade of liver stiffness and splenomegaly [9]. Higher platelet counts were reported in patients with nonalcoholic fatty liver disease (NAFLD) than in those with hepatitis $C$ for the same grade of liver stiffness [10]. However, no study has simultaneously evaluated the associated risk of thrombocytopenia in patients with CLD and the underlying etiology.

The distribution of patients among different CLD etiologies has been recently changing in Japan. Although hepatitis $B$ and $C$ remain the major causes of CLD, the percentages of patients have been decreasing; this is attributed to the implementation of screening programs and development of treatments [11]. Conversely, the percentages of patients with other causes of CLD, including nonalcoholic steatohepatitis (NASH) [11], NAFLD [11, 12], and alcoholic hepatitis [12, 13], have been increasing owing to lifestyle changes and an increase in disease recognition. Additionally, there are patients with CLD attributed to other causes, such as autoimmune hepatitis [12] and toxin/drug-induced hepatitis.

Investigating the differences in the development of thrombocytopenia among CLD etiologies is essential, considering the possibility of differences in the risk among different etiologies and recent changes in the distribution of patients. Therefore, we investigated the risk of decline in the platelet count and developing thrombocytopenia in an entire clinical course of CLD, including various states such as cirrhosis and cancer, across the different etiologies in a real-world setting in Japan.

\section{METHODS}

\section{Study Design and Data Source}

This was a claims-based cohort study. We conducted two analyses: a simulation with a random effects model [14] to estimate the changes in the platelet count (analysis 1) and a proportional hazard analysis [15] to examine the factors associated with the diagnosis of thrombocytopenia (analysis 2).

We used a health insurance claims database from April 2008 to August 2018, commercially provided by Medical Data Vision Co., Ltd. (Tokyo, Japan). The database consisted of data from acute care hospitals adopted by the Japanese Diagnosis and Procedure Combination (DPC) fixed-payment reimbursement system (i.e., DPC hospitals).

The database included approximately 25 million patients from 372 DPC hospitals, corresponding to approximately $22 \%$ of the total number of DPC hospitals in Japan as of August 2019 [16]. In Japan, approximately 30\% of all hospitals are DPC hospitals; they accounted for $>50 \%$ of the total number of beds as of April 2019. The database covered all patients receiving treatment in the hospitals regardless of the age and type of insurance and contained both in- and outpatient data. Furthermore, it comprised both claims and laboratory data, including the platelet count, of approximately $10 \%$ of the patients. This enabled us to investigate the changes in platelet counts based on the disease duration and assess the relationship between its baseline value and the incidence of thrombocytopenia. However, the number of times the data were collected and the time of collection of these data differed considerably among the patients. Thus, we performed a 
simulation to estimate the changes in the platelet count in analysis 1 . We also defined the incidence of thrombocytopenia based on a diagnosis of thrombocytopenia in analysis 2 . The study period included the total duration covered by the database.

This study was approved by the Ethics Committee of the University of Tokyo. All the procedures were in accordance with the Ethical Guidelines for Medical and Health Research Involving Human Subjects by the Ministry of Education, Culture, Sports, Science, and Technology and the Ministry of Health, Labour, and Welfare, Japan. The requirement for informed consent was waived because the database consisted of anonymized data collected for secondary use.

\section{Patient Identification}

Patients having at least one claim with a diagnosis of CLD based on the disease name recorded on the claim (see Supplementary material, Table S1) were defined as patients with CLD. These patients were classified into one of nine mutually exclusive categories: hepatitis $B$, hepatitis C, hepatitis B and C, viral hepatitis (virus not specified), autoimmune hepatitis, toxin/drug-induced hepatitis, alcoholic hepatitis, NASH, or other CLD, as shown in Fig. 1. In this classification, diagnoses indicating the cause of the disease were prioritized (those caused by viruses were attributed a higher priority than those caused by other factors) according to the diagnoses indicating the disease state. For example, if a patient had records of two different CLD diagnoses, where one was hepatitis $\mathrm{C}$ (categorized as hepatitis C) and the other was hepatocellular carcinoma (categorized as other CLD), the patient was categorized as having hepatitis C.

We extracted patients with CLD who had laboratory test data used as variables for each analysis. Patients with minimum one platelet count laboratory test were included in analysis 1. Patients with data on hemoglobin $(\mathrm{Hb}) \mathrm{A} 1 \mathrm{c}$, platelet count, alanine aminotransferase (ALT), and albumin testing within 12 weeks from the initial CLD diagnosis and without a thrombocytopenia diagnosis prior to the initial CLD diagnosis were included in analysis 2 .

\section{Statistical analysis}

In analysis 1 , we used a random effects model to assess the changes in the platelet count from the initial diagnosis of CLD (defined as the index date) according to the cause of CLD, as shown below:

$$
\begin{gathered}
\mathrm{PC}(i, t) \sim \operatorname{Normal}\left(\mu(i, t), \sigma^{2}\right) \\
\mu(i, t)=\beta_{0}+\beta_{1} t+\gamma_{i},
\end{gathered}
$$

where PC is the platelet count; $i$ corresponds to the identification number for each patient in the sample; $t$ represents the number of years from the index date; $\beta_{1}$ represents the change in the platelet count over time, the distribution of which was obtained using the Markov chain Monte Carlo method; $\gamma_{i}$ represents the random effects for each patient. The mean and 95\% credible interval of $\beta_{1}$ were calculated according to the cause of CLD to assess the changes in the platelet count in terms of the number of years from the index date. To study the effects of aging on the changes in the platelet counts, we plotted the mean platelet count at the initial diagnosis of CLD ( \pm 3 months) per age for patients with relevant test values. We obtained the change in the platelet count per year of age and then subtracted that from the value of $\beta_{1}$ to show marginal changes due to CLD.

In analysis 2, we performed a multivariate proportional hazard analysis. The day of the initial CLD diagnosis was defined as the index date. The incidence of thrombocytopenia was defined by a claim including a diagnosis termed as "thrombocytopenia," regardless of the availability or value of the platelet counts. The hazard ratio (HR) of the incidence of thrombocytopenia was calculated using the age at the index date, sex (1 for men; 2 for women), disease category (dummy variables, alcoholic hepatitis as reference group), and the HbA1c, platelet count, ALT, and albumin at baseline as explanatory variables. The baseline was defined as the period within 12 weeks before or after the 


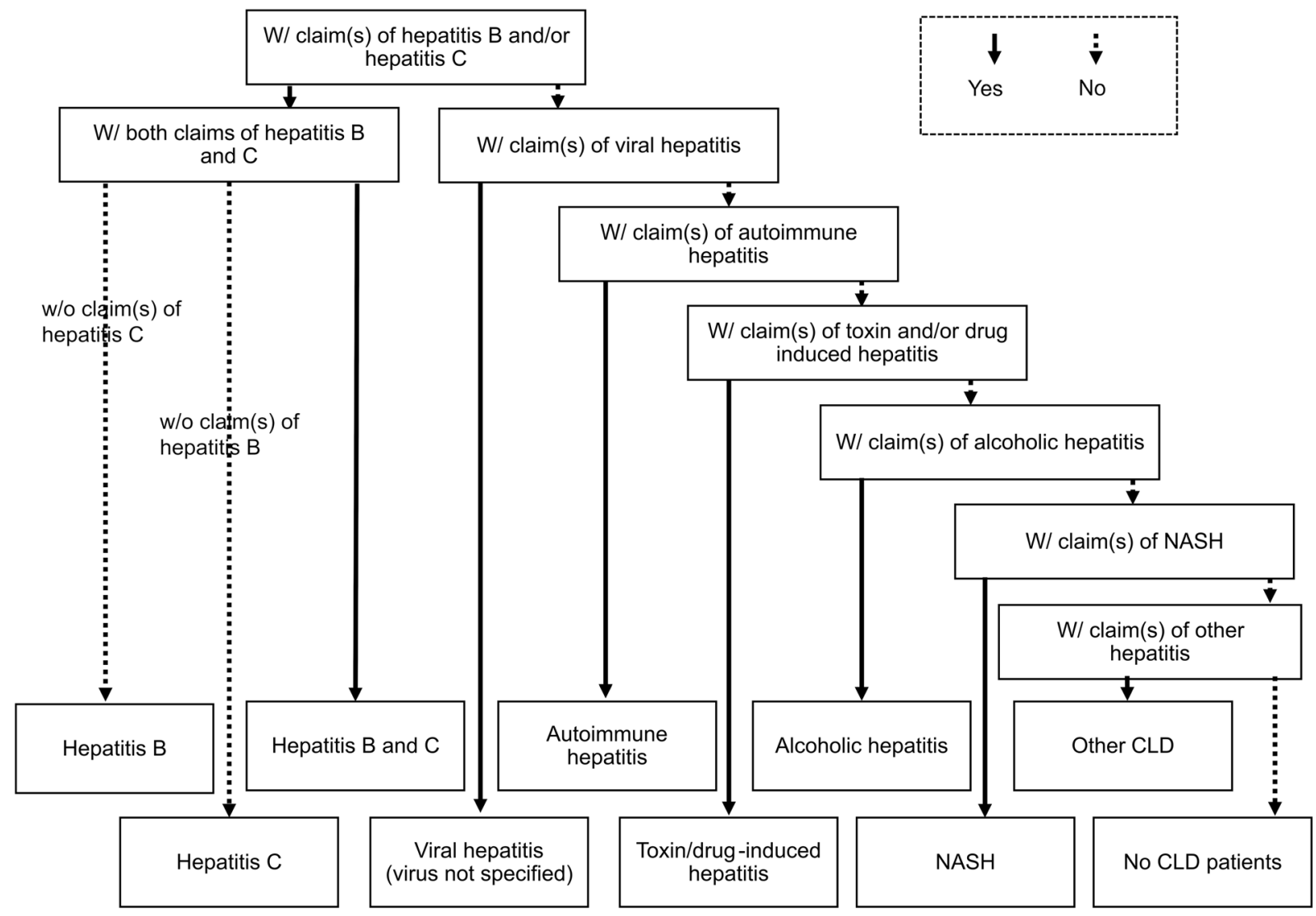

Fig. 1 Classification of patients with chronic liver disease for each disease category. Note: patients with more than one cause of CLD were classified into one category based

index date, and each value at baseline represented the value on the index date or the closest date to the index date. We considered HbA1c, ALT, and albumin as factors related to the CLD deterioration or severity; thus, we included them in the model. $P$-values $<0.05$ were considered statistically significant.

We used SAS version 9.4 (SAS Institute Inc., Cary, NC) and Microsoft Excel 2016 (Microsoft, Redmond, WA) software for the analyses.

\section{RESULTS}

\section{Patients}

A total of 675,699 patients with CLD (mean age, 62.8 years; women, $44.3 \%$ ) were included in the on this hierarchy between CLDs. CLD chronic liver disease, NASH nonalcoholic steatohepatitis, $w /$ with, $w / o$ without

database (Fig. 2). The number of patients identified for analyses 1 and 2 was 68,536 and 22,728 , respectively. Among the patients identified for analysis 1 , platelet count values were available for 47,781 patients at the initial diagnosis of CLD (see Fig. 2 for each disease category).

\section{Analysis 1: Decreased Platelet Count}

Figure 3 displays the mean platelet count at the initial diagnosis of CLD according to the patient age. The value decreased with age, and the decrease was estimated to be $0.88 \times 10^{9} / 1$ per year of age for ages 40-90 years.

The distribution of parameter $\beta_{1}$, estimating the changes in the platelet count over time for patients aged 40-79 years, according to the 


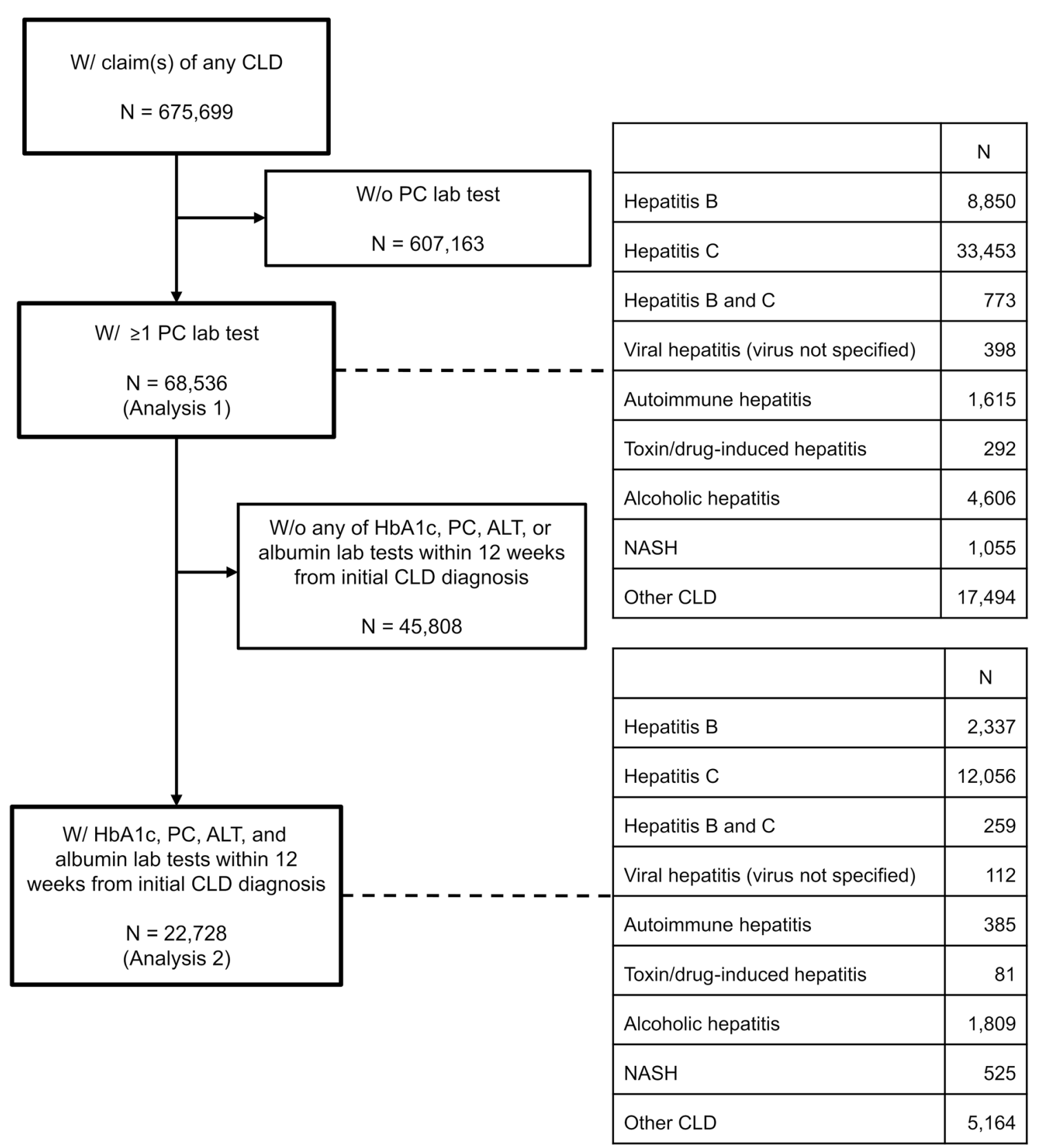

Fig. 2 Flowchart for patient identification. $A L T$ alanine aminotransferase, $A S T$ aspartate aminotransferase, $C L D$ chronic liver disease, $H b A 1 c$ hemoglobin A1c, NASH nonalcoholic steatohepatitis, $P T$ platelet count, $w /$ with, $w / o$ without

cause of CLD is shown in Table 1 (A. Before adjustment). The distribution of parameter $\beta_{1}$, after adjusting for the impact of aging on the platelet count is shown in Table 1 (B. After adjustment). The adjusted parameter $\beta_{1}$ was significantly negative, showing a significant reduction in the platelet count over time in the patients with hepatitis C (mean: $-0.75 \times 10^{9} / 1$ / year, $2.5 \%$ tile-97.5\%ile: $\quad-1.18 \times 10^{9} \quad$ to $-0.32 \times 10^{9} / 1 /$ year). The mean of parameter $\beta_{1}$ was negative; however, it was not significant in patients with hepatitis B $\left(-0.09 \times 10^{9} / 1 /\right.$ year, $-1.80 \times 10^{9}-1.63 \times 10^{9} / 1 /$ year $)$, toxin/drug-induced hepatitis $\left(-1.61 \times 10^{9} / 1 /\right.$ year, $-20.68 \times 10^{9}-19.01 \times 10^{9} / 1 /$ year $), \quad$ alcoholic hepatitis $\quad\left(-0.44 \times 10^{9} / 1 /\right.$ year, $\quad-5.64 \times$ 


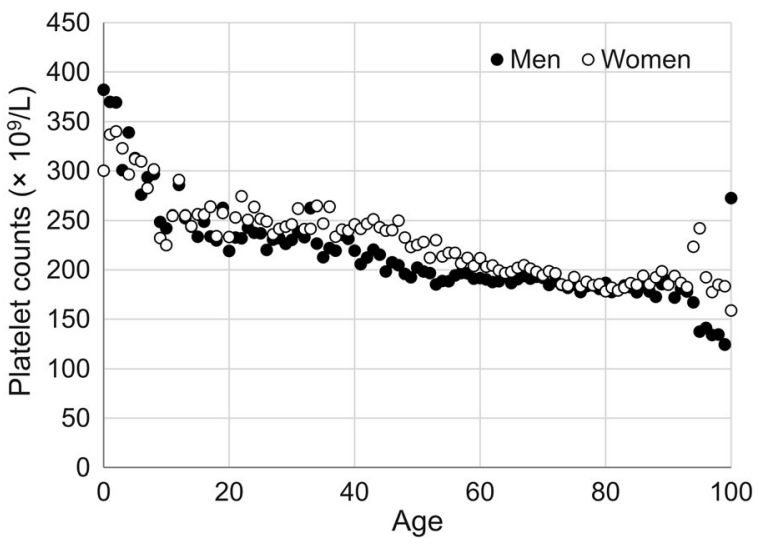

Fig. 3 Mean platelet counts on the date of initial diagnosis for chronic liver disease based on age

$\begin{array}{lcr}\left.10^{9}-4.58 \times 10^{9} / 1 / \text { year }\right), & \text { and } & \text { NASH } \\ \left(-2.38 \times 10^{9} / 1 / \text { year, }\right. & -10.60 \times 10^{9}-5.79 \times\end{array}$ $10^{9} / 1 /$ year).

\section{Analysis 2: Incidence of Thrombocytopenia}

Thrombocytopenia was detected in 708 of 22,728 patients (see Supplementary material, Table S2, regarding the mean platelet count per cause of CLD). The HR for the onset of thrombocytopenia was significantly lower for the patients with a higher baseline platelet count (HR, 0.873; $p<0.001$ ) and for those with a higher baseline albumin level (HR, 0.797; $p<0.001)$. No significant difference was observed in the patients with a higher baseline HbA1c (HR, 0.966; $p=0.235$ ) or ALT (HR, 1.066; $p=0.141$ ). A slightly and significantly lower HR was recorded for those with a higher age at the index (HR, 0.994; $p=0.037$ ), while no significant difference was observed between the sexes. Among the CLD etiologies, a higher HR was observed for patients with hepatitis $\mathrm{B}$ and $\mathrm{C}$ (HR, 2.992; $p<0.001)$, hepatitis B (HR, 2.879; $p<0.001)$, hepatitis C (HR, 1.876; $p<0.001)$, and other CLD (HR, 1.460; $p=0.012)$ than for patients with alcoholic hepatitis (Table 2).

\section{DISCUSSION}

We examined the changes in the platelet count over time according to the age and disease etiology and risk factors for thrombocytopenia in patients with CLD in a real-world setting in Japan. To the best of our knowledge, this is the first study to evaluate the association between the underlying etiology and other relevant parameters and the development of thrombocytopenia in patients with CLD using real-world data with a large number of patients.

We hypothesized that changes in the platelet count over time may be different among individuals owing to factors other than the etiology of CLD; thus, we employed a random effects model to estimate these differences. We also assumed that the changes due to aging should be considered when assessing the changes that occurred after the diagnosis of CLD. We found that the platelet count decreased with age; additionally, a significant decline in the platelet count over time was recorded in patients with hepatitis C. A non-significant decrease in platelet count was recorded in multiple CLD etiologies including hepatitis $\mathrm{B}$, toxin/druginduced hepatitis, alcoholic hepatitis, and $\mathrm{NASH}$, even after normalizing for the effects of aging. The incidence of thrombocytopenia was higher in patients with hepatitis B and/or C than in those with alcoholic hepatitis.

Thrombocytopenia is characterized by a multifactorial origin [2]. In patients with chronic hepatitis $\mathrm{C}$, multiple factors reportedly affect the pathogenesis of thrombocytopenia, including hypersplenism, a decrease in thrombopoietin levels, and suppression of platelet production in the bone marrow [17], which are also associated with other etiologies of CLD. Moreover, the hepatitis $\mathrm{C}$ virus induces immune-mediated platelet destruction involving antiplatelet antibodies and/or immune complex-associated platelet clearance [17]. Additionally, it has been suggested that if patients with CLD caused by hepatitis C have severe thrombocytopenia without a corresponding severity in liver disease, co-existing immune thrombocytopenic purpura should be suspected, which more often develops in patients with hepatitis $C$ than in healthy individuals and contributes to thrombocytopenia [17]. This multiplicity of factors may be related to the decline in the platelet count over time and significant risk of thrombocytopenia across 
Table 1 Mean and distribution of parameter $\beta_{1}$ per disease category before and after applying the change in platelet count per year of age

\begin{tabular}{|c|c|c|c|c|}
\hline \multirow[t]{2}{*}{ Description } & \multicolumn{4}{|c|}{$\beta_{1}\left(\times 10^{9} / 1 /\right.$ year $)$} \\
\hline & Mean & $2.5 \%$ ile & 50\%ile & $97.5 \%$ ile \\
\hline \multicolumn{5}{|l|}{ A. Before adjustment } \\
\hline Hepatitis B & -0.97 & -2.68 & -0.96 & 0.75 \\
\hline Hepatitis C & -1.63 & -2.07 & -1.62 & -1.20 \\
\hline Hepatitis B and C & 0.57 & -8.63 & 0.64 & 9.10 \\
\hline Viral hepatitis (virus not specified) & 10.50 & -1.22 & 10.64 & 21.62 \\
\hline Autoimmune hepatitis & -0.20 & -4.28 & -0.22 & 3.99 \\
\hline Toxin/drug-induced hepatitis & -2.49 & -21.56 & -2.58 & 18.12 \\
\hline Alcoholic hepatitis & -1.33 & -6.52 & -1.28 & 3.70 \\
\hline $\mathrm{NASH}$ & -3.26 & -11.48 & -3.33 & 4.91 \\
\hline Other CLD & -0.32 & -1.12 & -0.33 & 0.52 \\
\hline \multicolumn{5}{|l|}{ B. After adjustment } \\
\hline Hepatitis B & -0.09 & -1.80 & -0.08 & 1.63 \\
\hline Hepatitis C & -0.75 & -1.18 & -0.74 & -0.32 \\
\hline Hepatitis B and C & 1.45 & -7.75 & 1.52 & 9.99 \\
\hline Viral hepatitis (virus not specified) & 11.38 & -0.34 & 11.52 & 22.50 \\
\hline Autoimmune hepatitis & 0.68 & -3.40 & 0.66 & 4.87 \\
\hline Toxin/drug-induced hepatitis & -1.61 & -20.68 & -1.70 & 19.01 \\
\hline Alcoholic hepatitis & -0.44 & -5.64 & -0.40 & 4.58 \\
\hline NASH & -2.38 & -10.60 & -2.45 & 5.79 \\
\hline Other CLD & 0.56 & -0.24 & 0.55 & 1.40 \\
\hline
\end{tabular}

$C L D$ chronic liver disease, $N A S H$ nonalcoholic steatohepatitis

different etiologies observed in our study. Bone marrow suppression is also reportedly caused by other hepatitis viruses, including hepatitis A and $\mathrm{B}$ viruses, as well as by alcohol consumption and medications [4]. The decrease in the platelet count and the high risk of thrombocytopenia in patients with hepatitis $\mathrm{C}$ are reasonable when considering the variety of mechanisms. The high risk of thrombocytopenia and the decreasing tendency in platelet count in patients with hepatitis B may also be explained by mechanisms specific to hepatitis viruses.
For some causes of CLD, including toxin/drug-induced hepatitis, alcoholic hepatitis, and $\mathrm{NASH}$, a decreasing tendency of platelet count was recorded. However, although the baseline platelet count was adjusted in the proportional hazard analysis, no significant difference in the risk of thrombocytopenia was observed between alcoholic hepatitis and other causes of hepatitis.

The frequency of thrombocytopenia diagnosis may differ among CLD etiologies, even in patients with a similar platelet count owing to the differences in the frequency of outpatient visits or platelet count monitoring and CLD 
Table 2 Hazard ratio for the incidence of thrombocytopenia in multivariate proportional hazard analysis

\begin{tabular}{|c|c|c|c|}
\hline Parameter & $\begin{array}{l}\text { Parameter } \\
\text { estimate }\end{array}$ & $p$-value & HR \\
\hline Age at index & -0.006 & 0.037 & $0.994^{*}$ \\
\hline $\operatorname{Sex}^{\mathrm{a}}$ & -0.136 & 0.092 & 0.873 \\
\hline HbAlc (\%) & -0.035 & 0.235 & 0.966 \\
\hline $\operatorname{ALT}(\mathrm{U} / \mathrm{l})$ & 0.000 & 0.884 & 1.000 \\
\hline $\mathrm{PC}\left(10^{9} / \mathrm{l}\right)$ & -1.361 & $<0.001$ & $0.873^{*}$ \\
\hline Albumin (g/l) & -2.272 & $<0.001$ & $0.797^{*}$ \\
\hline Hepatitis $\mathrm{B}^{\mathrm{b}}$ & 1.057 & $<0.001$ & $2.879^{*}$ \\
\hline Hepatitis $C^{b}$ & 0.629 & $<0.001$ & $1.876^{*}$ \\
\hline Hepatitis $\mathrm{B}$ and $\mathrm{C}^{\mathrm{b}}$ & 1.096 & $<0.001$ & $2.992^{*}$ \\
\hline $\begin{array}{l}\text { Viral hepatitis (virus } \\
\text { not specified) }{ }^{\mathrm{b}}\end{array}$ & 0.193 & 0.789 & 1.212 \\
\hline $\begin{array}{c}\text { Autoimmune } \\
\text { hepatitis }^{\mathrm{b}}\end{array}$ & 0.308 & 0.371 & 1.361 \\
\hline $\begin{array}{l}\text { Toxin/drug-induced } \\
\text { hepatitis }\end{array}$ & 0.651 & 0.271 & 1.918 \\
\hline $\mathrm{NASH}^{\mathrm{b}}$ & -0.097 & 0.809 & 0.907 \\
\hline Other CLD ${ }^{b}$ & 0.379 & 0.012 & $1.460^{*}$ \\
\hline
\end{tabular}

$A L T$ alanine aminotransferase, CLD chronic liver disease, HbAlc hemoglobin Alc, NASH nonalcoholic steatohepatitis, $P C$ platelet count

${ }^{*} p<0.05$

${ }^{a}$ Sex: 1 for men; 2 for women

bisease category: dummy variables to alcoholic hepatitis as reference group

treatment. The risk of thrombocytopenia was associated with the baseline platelet count or baseline albumin in our proportional hazard analysis. However, although comorbid diabetes is reportedly a risk factor for CLD progression, particularly in NAFLD and NASH $[18,19]$, no significant risk of $\mathrm{HbA1c}$ with respect to thrombocytopenia was observed. Furthermore, no association was observed with a higher baseline ALT, although ALT increased in most CLDs [20]. Moreover, a lower risk was observed with a higher age at the index date, although the HR was 0.994. These results could be explained by the fact that the diagnosis of thrombocytopenia is attributed to patients requiring treatment to increase the platelet count and is not only based on the platelet count. Therefore, there could be many patients not diagnosed as having thrombocytopenia despite abnormally low platelet counts. Considering the decreasing tendency in the platelet count observed in most CLD etiologies, not only in patients with hepatitis $\mathrm{B}$ or $\mathrm{C}$ but also in those with non-viral hepatitis, continuous monitoring of the platelet count may be required and treatment should be considered if the platelet count is abnormally low.

This study has several limitations. First, the database included data from acute care hospitals; thus, patients with more severe CLDs may have been included when compared with all Japanese patients. Moreover, although the database included a large number of patients, those with laboratory testing represented only approximately $10 \%$ of the patients. This analysis included only patients with platelet count values; therefore, the number of eligible patients was limited, potentially introducing a selection bias. The differences in the sample size among patients with CLDs due to various etiologies may have affected the distribution of each value as well. Second, patients were classified into one of the several mutually exclusive categories of CLDs by prioritizing the diagnoses leading to CLD. However, if a patient's record had only a diagnosis indicating the disease state, such as hepatocellular carcinoma and hepatic cirrhosis, in the database, the patient was not classified into the category of the disease etiology but into that of other CLD. Nevertheless, we compared eight different CLDs based on the etiology. Third, in analysis 1, although the level of the platelet count at the index may be an indicator of disease progression, potentially affecting the change in platelet count later on, we compared the change in the platelet count among patients according to the cause of CLD, regardless of the platelet count at the index date. Fourth, since the study period included the entire database period (April 2008-August 2018), changes in the treatments, such as the advent of direct-acting antiviral 
agents in 2014, may have affected the results for certain causes of CLD. Fifth, as mentioned previously, we defined the incidence of thrombocytopenia based on the diagnoses in the claims data because not all the patients necessarily had records of platelet counts measured at a certain frequency. Moreover, this database was based on a single hospital for each patient; thus, diagnoses obtained in other settings were not included. Therefore, the number of patients with thrombocytopenia may have been underestimated because of the number of patients with abnormally low platelet counts. Nevertheless, our results may reflect a situation of thrombocytopenia diagnosis in a real-world setting. Finally, to estimate the effects of age on the change in the platelet count, populationbased data should be used; our dataset did not include population-based data. Consequently, we used the platelet count at the initial diagnosis of CLD, assuming that these patients would remain largely unaffected by the disease at this time. Although these limitations owing to the nature of the claims database study should be considered, we evaluated the risk of thrombocytopenia among CLD etiologies using two methods, based on the change in the platelet count and the diagnosis of thrombocytopenia in a real-world setting in Japan. We believe this study provides important information, considering the impact of thrombocytopenia for patients with CLD and recent changes in the distribution of patients among different CLD etiologies.

\section{CONCLUSION}

We illustrated the changes in the platelet counts and the risk factors for thrombocytopenia across different CLD etiologies using a nationwide claims database. The platelet counts tended to decrease with disease duration in most CLD categories; this decrease was particularly significant in patients with hepatitis C. The incidence of thrombocytopenia was more associated with hepatitis $\mathrm{B}$ and/or $\mathrm{C}$ than with alcoholic hepatitis. The frequency of thrombocytopenia diagnosis may differ among CLD etiologies, even in patients with similar platelet counts. Further research is warranted to explain the discrepancy between the decline in the platelet count and the diagnosis of thrombocytopenia, considering factors relevant to whether the diagnosis is made such as the frequency of outpatient visits and treatment of CLD.

\section{ACKNOWLEDGEMENTS}

We thank the participants of this study.

Funding. Sponsorship for this study, including the Journal's Rapid Service and Open Access fees, was provided by Shionogi \& Co., Ltd.

Medical Writing Assistance. Editorial assistance in the preparation of this article was provided by Editage (www.editage.com). Support for this assistance was funded by Shionogi $\&$ Co., Ltd.

Authorship. All named authors meet the International Committee of Medical Journal Editors (ICMJE) criteria for authorship for this article, take responsibility for the integrity of the work as a whole, and have given their approval for this version to be published.

Authors' Contributions. All authors contributed to the study design and interpretation of data. Manami Yoshida, Ryosuke Tateishi, Shinzo Hiroi, Masakazu Fujiwara, Yoshitake Kitanishi, and Ataru Igarashi contributed to the study conception. Kosuke Iwasaki contributed to the data analysis. Manami Yoshida and Tomomi Takeshima drafted the manuscript, and all authors revised it critically for important intellectual content. All authors approved the version to be published and agree to be accountable for all aspects of the work in ensuring that questions related to the accuracy or integrity of any part of the work are appropriately investigated and resolved.

Disclosures. Manami Yoshida, Shinzo Hiroi, and Yoshitake Kitanishi and his spouse are employees of Shionogi \& Co., Ltd. Ryosuke Tateishi declares no conflict of interest. 
Masakazu Fujiwara was an employee of Shionogi \& Co., Ltd., at the time the study was conducted. Masakazu Fujiwara is currently affiliated with AstraZeneca KK, Osaka, Japan. Kosuke Iwasaki and Tomomi Takeshima are employees of Milliman Inc., which has received consultancy fees from Shionogi \& Co., Ltd. Ataru Igarashi has received consulting fees and lecture fees from GSK Japan Inc., MSD Inc., Pfizer Japan Inc., Sanofi-Pasteur Inc., Novartis Pharma K.K., AbbVie GK, Astellas Pharma Inc., Chugai Pharmaceutical Co. Ltd., Asahi Kasei Pharma Inc., Novo Nordisk Pharma Inc., Eisai Inc., Beckton Dickinson and Company, Maruho Co. Ltd., Ono Pharmaceutical Inc., Sato Pharmaceutical Inc., Sumitomo Dainippon Pharma Inc., and Eli Lilly Japan K.K., outside the submitted work, and research grants from Taiho Pharmaceutical Co. Ltd., GSL Behring Japan Inc., Gilead Science K.K., Fuji Film K.K., Terumo Corporation, Takeda Pharmaceutical Inc., Boston Scientific Japan Inc., DeSC Healthcare Inc., Otsuka Pharmaceutical K.K., Varian Medical Systems Inc., and Intuitive Surgical GK., outside the submitted work.

Compliance with Ethics Guidelines. This study was approved by Ethical Committee of the University of Tokyo. All procedures were in accordance with the Ethical Guidelines for Medical and Health Research Involving Human Subjects by the Ministry of Education, Culture, Sports, Science, and Technology and the Ministry of Health, Labour, and Welfare, Japan. Informed consent was waived because the database consisted of anonymized data that had been collected for secondary use.

Prior Presentation. Parts of this work were presented at the International Liver Congress 2019, October 1-2, 2019, Osaka, Japan.

Data Availability. The datasets analyzed during the current study are available from the authors upon reasonable request and with permission of Medical Data Vision Co., Ltd.

Open Access. This article is licensed under a Creative Commons Attribution-NonCommercial 4.0 International License, which permits any non-commercial use, sharing, adaptation, distribution and reproduction in any medium or format, as long as you give appropriate credit to the original author(s) and the source, provide a link to the Creative Commons licence, and indicate if changes were made. The images or other third party material in this article are included in the article's Creative Commons licence, unless indicated otherwise in a credit line to the material. If material is not included in the article's Creative Commons licence and your intended use is not permitted by statutory regulation or exceeds the permitted use, you will need to obtain permission directly from the copyright holder. To view a copy of this licence, visit http://creativecommons.org/licenses/by$\mathrm{nc} / 4.0 /$.

\section{REFERENCES}

1. Afdhal N, McHutchison J, Brown R, et al. Thrombocytopenia associated with chronic liver disease. J Hepatol. 2008;48:1000-7.

2. Peck-Radosavljevic M. Thrombocytopenia in chronic liver disease. Liver Int. 2017;37:778-93.

3. Giannini EG. Review article: thrombocytopenia in chronic liver disease and pharmacologic treatment options. Aliment Pharmacol Ther. 2006;23: 1055-65.

4. Mitchell O, Feldman DM, Diakow M, Sigal SH. The pathophysiology of thrombocytopenia in chronic liver disease. Hepat Med. 2016;8:39-50.

5. Bashour FN, Teran JC, Mullen KD. Prevalence of peripheral blood cytopenias (hypersplenism) in patients with nonalcoholic chronic liver disease. Am J Gastroenterol. 2000;95:2936-9.

6. Ferrara J, Ellison EC, Martin EW Jr, Cooperman M. Correction of hypersplenism following distal splenorenal shunt. Surgery. 1979;86:570-3.

7. McAllister E, Goode S, Cordista AG, Rosemurgy A. Partial portal decompression alleviates thrombocytopenia of portal hypertension. Am Surg. 1995;61: 129-31.

8. Miller JB, Figueroa EJ, Haug RM, Shah NL. Thrombocytopenia in chronic liver disease and the role of thrombopoietin agonists. Gastroenterol Hepatol. 2019; 15:326-32. 
9. Tejima K, Masuzaki R, Ikeda $\mathrm{H}$, et al. Thrombocytopenia is more severe in patients with advanced chronic hepatitis $C$ than $B$ with the same grade of liver stiffness and splenomegaly. J Gastroenterol. 2010;45:876-84.

10. Mawatari H, Yoneda M, Kirikoshi H, Maeda S, Nakajima A, Saito S. Thrombocytopenia is more severe in patients with chronic hepatitis $C$ than in patients with nonalcoholic fatty liver disease. J Gastroenterol. 2012;47(5):606-7.

11. Sarin SK, Kumar M, Eslam M, et al. Liver diseases in the Asia-Pacific region: a Lancet Gastroenterology \& Hepatology Commission [published correction appears in Lancet Gastroenterol Hepatol. 2020 Mar; 5(3):e2]. Lancet Gastroenterol Hepatol. 2020;5: $167-228$.

12. Tateishi R, Okanoue T, Fujiwara N, et al. Clinical characteristics, treatment, and prognosis of non- $\mathrm{B}$, non-C hepatocellular carcinoma: a large retrospective multicenter cohort study. J Gastroenterol. 2015;50:350-60.

13. Horie Y, Ebinuma H, Kanai T. Current status of alcoholic liver diseases in Japan. Nippon Shokakibyo Gakkai Zasshi. 2015;112:1630-40.
14. Laird NM, Ware JH. Random-effects models for longitudinal data. Biometrics. 1982;38:963-74.

15. Cox DR. Regression models and life-tables. J R Stat Soc Series B Stat Methodol. 1972;34:187-220.

16. Pharmacoepidemiology \& Database Taskforce, Japanese Society for Pharmacoepidemiology. Survey of Japanese databases in Japan available for clinical/pharmacoepidemiology. 2020. http://www. jspe.jp/committee/020/0210/. Accessed 12 Oct 2020.

17. Weksler BB. Review article: the pathophysiology of thrombocytopenia in hepatitis $\mathrm{C}$ virus infection and chronic liver disease. Aliment Pharmacol Ther. 2007;26:13-9.

18. Adams L. Nonalcoholic fatty liver disease and diabetes mellitus. Endocr Res. 2007;32:59-69.

19. Moscatiello S, Manini R, Marchesini G. Diabetes and liver disease: an ominous association. Nutr Metab Cardiovasc Dis. 2007;17:63-70.

20. Johnston DE. Special considerations in interpreting liver function tests. Am Fam Phys. 1999;59: 2223-30. 